TRANSACTIONS OF THE

AMERICAN MATHEMATICAL SOCIETY

Volume 356, Number 5, Pages 2049-2066

S 0002-9947(03)03531-1

Article electronically published on December 12, 2003

\title{
SPINORS AS AUTOMORPHISMS OF THE TANGENT BUNDLE
}

\author{
ALEXANDRU SCORPAN
}

\begin{abstract}
We show that, on a 4 -manifold $M$ endowed with a spin ${ }^{\mathbb{C}}$-structure induced by an almost-complex structure, a self-dual (positive) spinor field $\phi \in \Gamma\left(W^{+}\right)$is the same as a bundle morphism $\phi: T_{M} \rightarrow T_{M}$ acting on the fiber by self-dual conformal transformations, such that the Clifford multiplication is just the evaluation of $\phi$ on tangent vectors, and that the squaring map $\sigma: \mathcal{W}^{+} \rightarrow \Lambda^{+}$acts by pulling-back the fundamental form of the almostcomplex structure. We use this to detect Kähler and symplectic structures.
\end{abstract}

\section{INTRODUCTION}

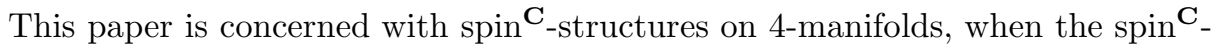
structure is induced from an almost-complex structure. The aim of the paper is two-fold. On the one hand, we will present a non-standard language for describing self-dual (= positive) spinor fields as automorphisms of the tangent bundle. On the other hand, using spinor fields to deform Hermitian structures, we detect Kähler structures (Theorem 1.2) and characterize symplectic structures (Corollary 1.3), expanding upon results from Sco02].

Let $M$ be an oriented 4-manifold, endowed with a metric $g$. Using this metric, we can identify $g$-orthogonal almost-complex structures with self-dual 2-forms of constant length $\sqrt{2}$. Thus, for example, for a non-zero self-dual 2-form $\alpha$, we can define its Chern class $c_{1}(\alpha)$ as the first Chern class of the associated almost-complex structure.

Choose an almost-complex structure $\omega$. It induces a standard $\operatorname{spin}^{\mathrm{C}_{\text {-structure }}}$ with spinor bundles denoted $\mathcal{W}^{ \pm}$, with determinant bundle $K^{*}=\operatorname{det}_{\mathbb{C}} \mathcal{W}^{ \pm}=$ $\operatorname{det}_{\mathbb{C}}\left(T_{M}, \omega\right)$, and with a Clifford multiplication denoted by $T_{M} \times \mathcal{W}^{+} \stackrel{\bullet}{\longrightarrow} \mathcal{W}^{-}$. It is a standard fact that $\mathcal{W}^{-} \approx\left(T_{M}, \omega\right)$ as complex bundles. A choice of unitary connection $A$ on $K^{*}$, together with the Levi-Cività connection $\nabla$ of $g$, induces unique unitary connections $\bar{\nabla}^{A}$ on $\mathcal{W}^{+}$and $\widetilde{\nabla}^{A}$ on $\mathcal{W}^{-}$, related by

$$
\widetilde{\nabla}^{A}(v \bullet \phi)=(\nabla v) \bullet \phi+v \bullet\left(\bar{\nabla}^{A} \phi\right) .
$$

An important related object is the associated Dirac operator $\mathcal{D}^{A}: \Gamma\left(\mathcal{W}^{+}\right) \rightarrow$ $\Gamma\left(\mathcal{W}^{-}\right)$, defined by $\mathcal{D}^{A} \phi=\sum e_{k} \bullet \bar{\nabla}_{e_{k}}^{A} \phi$ for any $g$-orthonormal frame $\left\{e_{k}\right\}$. Another important object is the quadratic map $\sigma: \mathcal{W}^{+} \rightarrow \Lambda^{+}$, which is famous for appearing in the Seiberg-Witten equations, but see also LM89, IV.10].

A section of $\mathcal{W}^{+}$will be called a self-dual spinor field. (A more customary terminology would be "positive spinor field". We prefer to say "self-dual spinor

Received by the editors April 24, 2002 and, in revised form, April 15, 2003.

2000 Mathematics Subject Classification. Primary 53C27; Secondary 57N13, 32Q60, 53D05.

Key words and phrases. Spinor, four-manifold, almost-complex, symplectic, Kähler. 
field", which is used in the classical paper [AHS78] and seems better suited to the peculiarities of dimension 4 and to the phenomena described in this paper.)

A first aim of this paper is to show that self-dual spinor fields $\phi \in \Gamma\left(\mathcal{W}^{+}\right)$can be identified with self-dual conformal transformations

$$
\phi: T_{M} \rightarrow T_{M},
$$

i.e. with bundle maps that act on each fiber by rotating a pair of orthogonal planes by the same angle, in directions compatible with the orientation of $M$, and then dilate/shrink the fiber by multiplying with a scalar. This bundle identification can be easily obtained from the starting steps of Tau95, Tau00 (see Remark 2.3), but we strengthen it by noticing that the Clifford multiplication identifies with the evaluation, as

$$
v \bullet \phi \equiv \phi(v)
$$

and that the quadratic map $\sigma: \mathcal{W}^{+} \rightarrow \Lambda^{+}$can be described as giving the pull-back of the almost-complex form, as

$$
\sigma(\phi) \equiv \frac{1}{4} \phi^{*} \omega
$$

where $\left(\phi^{*} \omega\right)(v, w)=\omega(\phi v, \phi w)$. This non-standard language is stated in Theorem 2.1.

Thus, one can use spinor fields to deform almost-complex structures (within a Chern class; see 2.5).

A second aim of this paper is to expand on the following result:

Proposition 1.1 ([Sc002]). Consider a 4-manifold $M$ endowed with a metric $g$ and with the spin ${ }^{\mathrm{C}}$-structure induced from an almost-complex structure $\omega$. Assume that $H^{2}(M ; \mathbb{Z})$ has no 2-torsion. Then the equality $\alpha=\sigma(\phi)$ establishes a bijection between:

(A) The set of all Kähler forms $\alpha$ with $c_{1}(\alpha)=c_{1}(\omega)$ and compatible with a metric scalar-multiple of $g$; and the set of all gauge classes of pairs $(\phi, A)$ with $\phi$ nowhere-zero and $\bar{\nabla}^{A} \phi=0$.

(B) The set of all symplectic forms $\alpha$ with $c_{1}(\alpha)=c_{1}(\omega)$ and compatible with a metric conformal to $g$; and the set of all gauge classes of pairs $(\phi, A)$ with $\phi$ nowhere-zero, $\mathcal{D}^{A} \phi=0$, and $\left\langle\bar{\nabla}^{A} \phi, i \phi\right\rangle_{\mathbb{R}}=0$.

(Here, "gauge class" means equivalent with respect to the action of the gauge group $\mathcal{G}=\left\{f: M \rightarrow \mathbb{S}^{1}\right\}$ of $K^{*}$. It acts on $K^{*}$ and $\mathcal{W}^{ \pm}$by scalar multiplication, and that induces an action on unitary connections on $K^{*}$ and on sections of $\mathcal{W}^{ \pm}$ (and thus on pairs $(\phi, A)$ ). See also Remark 2.5])

An immediate remark about Proposition 1.1 is the lack of symmetry of (A) and (B): one statement deals with metrics scalar-multiple of $g$, the other with metrics conformal to $g$. Another is that the term $\left\langle\bar{\nabla}^{A} \phi, i \phi\right\rangle_{\mathbb{R}}$, while formally clear, has a rather obscure intuitive meaning. These remarks will be addressed as follows:

We will extend statement (A) from above to include all Kähler forms compatible with metrics conformal to $g$ (instead of merely scalar-multiple of $g$ ). But the connections considered so far are not enough. We need a more general set of connections $\widetilde{\nabla}$ on $\mathcal{W}^{-}$(called "admissible connections") that do not relate to any connections $A$ on $K^{*}$, and do not correspond (via (I)) to connections $\bar{\nabla}$ on $\mathcal{W}^{+}$, but to connections $\bar{\nabla}$ on the larger bundle $\operatorname{Hom}\left(T_{M}, T_{M}\right) \supset \mathcal{W}^{+}$. 
Concretely, using the Hermitian isomorphism $\mathcal{W}^{-} \approx T_{M}$, a connection $\widetilde{\nabla}$ on $\mathcal{W}^{-}$ is admissible if it is $\mathbb{C}$-linear for $\omega$ and $g$-metric. (The connections $\widetilde{\nabla}^{A}$ associated to connections $A$ on $K^{*}$ need the extra condition $\left.\widetilde{\nabla}^{A}\right|_{\Lambda^{-}}=\left.\nabla\right|_{\Lambda^{-}}$; see Lemma [3.1) An admissible connection $\widetilde{\nabla}$ defines a connection $\bar{\nabla}$ on $\operatorname{Hom}\left(T_{M}, T_{M}\right)$ through $(\bar{\nabla} \phi)(v)=\widetilde{\nabla}(\phi v)-\phi(\nabla v)$. The latter is simply a version of (1) read using (2), and is natural if we view $\phi:\left(T_{M}, \nabla\right) \rightarrow\left(T_{M}, \widetilde{\nabla}\right)$.

The extension of 1.1(A) is:

Theorem 1.2. Assume $H^{2}(M ; \mathbb{Z})$ has no 2-torsion. The equality $\alpha=\sigma(\phi)$ establishes a bijection between: the set of all Kähler forms $\alpha$ with $c_{1}(\alpha)=c_{1}(\omega)$ and compatible with a metric conformal to $g$; and the set of all gauge classes of pairs $(\phi, \widetilde{\nabla})$ with $\widetilde{\nabla}$ admissible, $\phi$ nowhere-zero, and with

$$
\left(\bar{\nabla}_{X} \phi\right)(Y)=\left(\bar{\nabla}_{Y} \phi\right)(X) .
$$

(Here again, the gauge group $\mathcal{G}$ acts by scalar multiplication on $\mathcal{W}^{-}=T_{M}$ and thus induces an action on connections $\widetilde{\nabla}$, and hence on pairs $(\phi, \widetilde{\nabla})$. See also Remark[2.5])

Theorem 1.2 above could be read as a strong 4-dimensional $\operatorname{spin}^{\mathrm{C}}$ cousin of Proposition 9.10 from LM89 p. 340]. The latter states: Let $M^{n}$ be endowed with a spin-structure, and let $\phi$ be a pure spinor. Then $\phi$ determines an integrable almostcomplex structure if and only if $Y \bullet \bar{\nabla}_{X} \phi=X \bullet \bar{\nabla}_{Y} \phi$, for all $X, Y$ from the kernel of the map $T_{M} \otimes \mathbb{C} \rightarrow \mathcal{W}, w \mapsto w \bullet \phi$, and where $\vec{\nabla}$ is the unique spin-connection on the spinor bundle $\mathcal{W}$. Notice that this statement mentions only integrability, but not Kähler.

This paper will also interpret the rather mysterious term $\left\langle\bar{\nabla}^{A} \phi, i \phi\right\rangle_{\mathbb{R}}$ from Proposition 1.1(B). It appeared there due to the formula

$$
\|\phi\|^{2} \mathcal{D}^{A} \phi=i\left(2 d^{*} \sigma(\phi)+\left\langle\bar{\nabla}^{A} \phi, i \phi\right\rangle_{\mathbb{R}}\right) \bullet \phi
$$

from $[\mathrm{ScO02}$.

We will show that the 1 -form $\left\langle\bar{\nabla}^{A} \phi, i \phi\right\rangle_{\mathbb{R}}$ measures how close are the connections $\nabla$ and $\widetilde{\nabla}^{A}$ when compared through $\phi:\left(T_{M}, \nabla\right) \rightarrow\left(T_{M}, \widetilde{\nabla}^{A}\right)$ (see Lemma 3.9). In standard terms, $\left\langle\bar{\nabla}^{A} \phi, i \phi\right\rangle$ measures how far $\bar{\nabla}^{A} \phi$ is from being minimal (when $A$ varies). (That is, $\left\langle\bar{\nabla}^{A} \phi, i \phi\right\rangle=0$ if and only if $\bar{\nabla}^{A} \phi$ has pointwise minimal length.)

Underlying the above discussion is the general comparison of $\nabla$ and $\widetilde{\nabla}^{A}$ via $\phi$. It is governed by an analogue of a "second fundamental form":

$$
\left(\mathcal{B}^{A} \phi\right)_{X} Y=\widetilde{\nabla}_{X}^{A}(\phi Y)-\phi\left(\nabla_{X} Y\right)
$$

In standard terms, it is simply

$$
\left(\mathcal{B}^{A} \phi\right)_{X} Y=Y \bullet \bar{\nabla}_{X}^{A} \phi
$$

It naturally splits as

$$
\mathcal{B}^{A} \phi=\operatorname{Alt} \mathcal{B}^{A} \phi+\operatorname{Sym}_{0} \mathcal{B}^{A} \phi+g \otimes \frac{1}{4} \mathcal{D}^{A} \phi
$$

where Alt $\mathcal{B}$ is the alternating (skew-symmetric) part, $\operatorname{Sym}_{0} \mathcal{B}$ is the tracelesssymmetric part, and $g \otimes \frac{1}{4} \mathcal{D}^{A} \phi$ is the trace part of $\mathcal{B}$.

The skew-symmetric part Alt $\mathcal{B}$ compares through $\phi$ the torsions of $\nabla$ and $\widetilde{\nabla}$, and underlies Theorem 1.2 above. Namely, Alt $\mathcal{B} \phi=0$ means $\left(\bar{\nabla}_{X} \phi\right)(Y)=\left(\bar{\nabla}_{Y} \phi\right)(X)$, and in that case $\sigma(\phi)=\frac{1}{4} \phi^{*} \omega$ is Kähler for $\phi^{*} g$. On the other hand, the symmetric part $\operatorname{Sym} \mathcal{B}$ can be understood as the linear extension of terms like $\left(\mathcal{B}^{A} \phi\right)_{X} X$, which 
compare geodesics in the $X$-direction. The trace $\mathcal{D}^{A} \phi$ can thus be understood as an average comparison of geodesics.

As mentioned, $\left\langle\bar{\nabla}^{A} \phi, i \phi\right\rangle$ vanishes exactly when $\mathcal{B}^{A} \phi$ is pointwise minimized. Trying to minimize the other terms appearing in the splitting (4) through variation of $A$ among unitary connections on $K^{*}$, we will show that the various minimizing connections must all sit on a single affine line and are distanced at fixed ratios (see Theorem 3.7). Therefore, if two such minimizing connections happen to coincide, then all of them must coincide.

The conditions from 1.1 (B) can be read now as follows: both $\mathcal{B}^{A} \phi$ and $\mathcal{D}^{A} \phi$ are minimized by the same $A$. But then this $A$ must minimize all terms from (4). In particular it must minimize $\operatorname{Alt}^{A} \phi$, that is, minimize the torsion of the connection $\widetilde{\nabla}^{A}$ when viewed through $\phi$.

In conclusion, Proposition 1.1(B) can be rephrased as: The form $\sigma(\phi)=\frac{1}{4} \phi^{*} \omega$ is symplectic if and only if there is a connection $A$ that simultaneously minimizes all components of $\mathcal{B}^{A} \phi=\bar{\nabla}^{A} \phi$.

In particular, we have: The form $\phi^{*} \omega$ is symplectic if and only if there is a connection $\widetilde{\nabla}^{A}$ on $T_{M}$ which, viewed through $\phi:\left(T_{M}, \nabla\right) \rightarrow\left(T_{M}, \widetilde{\nabla}^{A}\right)$, simultaneously is a closest match to $\nabla$ (i.e. $\mathcal{B}^{A} \phi$ minimal) and has the torsion minimized (i.e. Alt $\mathcal{B}^{A} \phi$ minimal). Or even:

Corollary 1.3. The manifold $(M, g, \omega)$ is almost-Kähler if and only if there is a $\mathbb{C}$-linear g-metric connection $\widetilde{\nabla}$ on $T_{M}$ with $\left.\widetilde{\nabla}\right|_{\Lambda^{-}}=\left.\nabla\right|_{\Lambda^{-}}$, and that simultaneously is closest to $\nabla$ and has minimal torsion.

We hope this characterization might shed some new light on the nature of almost-complex structures compatible with symplectic structures.

In what follows, Section 2 will deal with proving the non-standard language for spinors, while Section 3 will detail its geometric ramifications.

\section{DiCTIONARY}

Let $M$ be a closed oriented 4-manifold, endowed with a fixed Riemannian metric $g$ and its Levi-Cività connection $\nabla$. The same notation " $\nabla$ " will denote the connections induced by $\nabla$ on the tensor bundles of $M$. Using the metric, we will systematically identify $T_{M}$ and $T_{M}^{*}$, and their corresponding bundles of tensors, including $\Lambda\left(T_{M}\right)$ and $\Lambda\left(T_{M}^{*}\right)$. Throughout the paper, a suddenly appearing " $x$ " will simply mean a generic point of $M$.

An almost-complex structure is an automorphism $J: T_{M} \rightarrow T_{M}$ such that $J \circ J=$ $-i d$. All almost-complex structures considered will be compatible with the chosen orientation of $M$, and will be $g$-orthogonal, i.e. $g(v, w)=g(J v, J w)$. The metric allows us to identify all such almost-complex structures $J$ with self-dual 2-forms $\omega \in \Gamma\left(\Lambda^{+}\right)$of constant length $\sqrt{2}$ such that $\omega(v, w)=g(J v, w)$. (This is just a particular instance of the isomorphism $\Lambda^{2}\left(\mathbb{R}^{4}\right) \approx \mathfrak{s o}(4)$, identifying 2-forms with skew-symmetric endomorphisms.) The complex-line bundle $K^{*}=\operatorname{det}_{\mathbb{C}}\left(T_{M}, \omega\right)$ is called the anti-canonical bundle of $(M, \omega)$. We denote by $c_{1}(\omega)$ the Chern class $c_{1}\left(K^{*}\right)=c_{1}\left(T_{M}, \omega\right)$.

A non-degenerate 2 -form $\omega$ with $d \omega=0$ is called symplectic. If a symplectic form is self-dual and of constant length $\sqrt{2}$, then we call it a symplectic form compatible with the metric $g$ (and in that case $(M, g, \omega)$ is an almost-Kähler manifold). A 
self-dual 2-form $\omega$ of length $\sqrt{2}$ such that $\nabla \omega=0$ will be called a Kähler form compatible with $g$ (since $(M, g, \omega)$ is a Kähler manifold).

The method we choose for proving Theorem 2.1 below employs quaternions, and is inspired by the exposition from [Akb96].

Denote by $\mathbb{H}$ the division algebra of quaternions, and by $\mathbb{S}^{3}$ its unit sphere. The choice of any isomorphism $\mathbb{H} \approx \mathbb{R}^{4}$ that preserves orientation and inner product allows us to identify $S O(4)=\mathbb{S}^{3} \times \mathbb{S}^{3} / \pm 1$ acting on $\mathbb{R}^{4}$ by

$$
S O(4) \times \mathbb{R}^{4} \longrightarrow \mathbb{R}^{4} \quad: \quad\left[\xi_{+}, \xi_{-}\right] \cdot v=\xi_{+} v \xi_{-}^{-1} .
$$

If we further identify $\mathbb{H}$ with $\mathbb{C}^{2}$, through $z_{1}+z_{2} j \equiv\left(z_{1}, z_{2}\right)$, then we can identify $S U(2)=\mathbb{S}^{3}$ acting by

$$
S U(2) \times \mathbb{C}^{2} \longrightarrow \mathbb{C}^{2} \quad: \quad \xi \cdot v=v \xi^{-1}
$$

The full unitary group can be identified as $U(2)=\mathbb{S}^{1} \times \mathbb{S}^{3} / \pm 1$ acting on $\mathbb{C}^{2}$ by

$$
U(2) \times \mathbb{C}^{2} \longrightarrow \mathbb{C}^{2} \quad: \quad[\lambda, \xi] \cdot v=\lambda v \xi^{-1} .
$$

The complex-spin group is $\operatorname{Spin}^{\mathbb{C}}(4)=\mathbb{S}^{1} \times \mathbb{S}^{3} \times \mathbb{S}^{3} / \pm 1$.

Since $M$ is oriented and endowed with a metric, its tangent bundle $T_{M}$ admits a defining cocycle with values in $S O(4)$. That means that there is a covering of $M$ by open sets $\left\{U_{\gamma}\right\}$ and a collection of transition maps $\left\{\tau_{\alpha \beta}: U_{\alpha} \cap U_{\beta} \rightarrow S O(4)\right\}$ such that the bundle $T_{M} \rightarrow M$ can be obtained by gluing trivial-bundle pieces $U_{\gamma} \times \mathbb{R}^{4} \rightarrow U_{\gamma}$ through the identification of $\left(x, v_{\alpha}\right) \in U_{\alpha} \times \mathbb{R}^{4}$ with $\left(x, v_{\beta}\right) \in U_{\beta} \times \mathbb{R}^{4}$ when $v_{\alpha}=\tau_{\alpha \beta}(x) v_{\beta}$.

A spin ${ }^{\mathbf{C}}$-structure on $M$ is (the equivalence class of) a lifting of the cocycle $\left\{\tau_{\alpha \beta}\right\}$ to a cocycle $\left\{\widetilde{\tau}_{\alpha \beta}\right\}$ with values in $\operatorname{Spin}^{\mathbb{C}}(4)$, lifted via the natural map

$$
\operatorname{Spin}^{\mathbb{C}}(4) \longrightarrow S O(4) \quad: \quad\left[\lambda, \xi_{+}, \xi_{-}\right] \longmapsto\left[\xi_{+}, \xi_{-}\right] .
$$

A choice of such a lifted cocycle $\left\{\widetilde{\tau}_{\alpha \beta}\right\}$ induces, through the two maps

$$
\operatorname{Spin}^{\mathbb{C}}(4) \longrightarrow U(2) \quad: \quad\left[\lambda, \xi_{+}, \xi_{-}\right] \longmapsto\left[\lambda, \xi_{ \pm}\right]
$$

defining $U(2)$-cocycles for two complex-plane bundles $\mathcal{W}^{+}$and $\mathcal{W}^{-}$, called the bundles of self-dual and anti-self-dual spinors (or "positive" and "negative spinors"). Through the map $\operatorname{Spin}^{\mathbb{C}}(4) \rightarrow \mathbb{S}^{1},\left[\lambda, \xi_{+}, \xi_{-}\right] \longmapsto \lambda^{2}$, the cocycle $\left\{\widetilde{\tau}_{\alpha \beta}\right\}$ also induces a defining cocycle for the complex line bundle $L=\operatorname{det}_{\mathbb{C}} \mathcal{W}^{ \pm}$, which is called the determinant line bundle of the $\operatorname{spin}^{\mathrm{C}}$-structure. Every 4-manifold admits at least one spin ${ }^{C_{-}}$-structure.

The spinor bundles also come equipped with a Clifford multiplication $T_{M} \times$ $\mathcal{W}^{+} \stackrel{\bullet}{\longrightarrow} \mathcal{W}^{-}$(and its adjoint $T_{M} \times \mathcal{W}^{-} \rightarrow \mathcal{W}^{+}$), characterized by the property that

$$
v \bullet(v \bullet \phi)=-\|v\|^{2} \phi
$$

This is in fact part of an action of the complexified Clifford algebra bundle $\mathrm{C} \ell\left(T_{M}\right) \otimes$ $\underline{\mathbb{C}}$ on $\mathcal{W}^{+} \oplus \mathcal{W}^{-}$(see [LM89, Ch. II] for details). Since as vector bundles $\mathrm{C} \ell\left(T_{M}\right) \approx$ $\bar{\Lambda}\left(T_{M}\right)$, there is an induced Clifford action of $\Lambda^{+}\left(T_{M}\right)$ on $\mathcal{W}^{+}$.

It is known that, via Clifford multiplication, we have $\operatorname{End}_{0}\left(\mathcal{W}^{+}\right) \approx \Lambda^{+} \otimes \mathbb{C}$ (where $\operatorname{End}_{0}$ denotes the set of traceless $\mathbb{C}$-endomorphisms). On the other hand, for every $\phi \in \mathcal{W}^{+}$, consider the endomorphism $\phi \otimes \phi^{*}=\langle\cdot, \phi\rangle \phi$ of $\mathcal{W}^{+}$. Its traceless part is $\phi \otimes \phi^{*}-\frac{1}{2}\|\phi\|^{2} i d$. The latter corresponds to an element of $\Lambda^{+} \otimes \mathbb{C}$, which 
turns out to be purely imaginary, i.e. of the form $i \sigma(\phi)$ for some $\sigma(\phi) \in \Lambda^{+}$. This defines the squaring map

$$
\sigma: \mathcal{W}^{+} \rightarrow \Lambda^{+} .
$$

Alternatively, $\sigma$ is uniquely characterized by its codomain and the property

$$
\sigma(\phi) \bullet \phi=-i \frac{\|\phi\|^{2}}{2} \phi .
$$

This map is involved in the Seiberg-Witten equations, see for example [Wit94, Don96, Mor96.

If $(M, g)$ is endowed with a compatible almost-complex structure $\omega$, then the cocycle of $T_{M}$ can be reduced to a cocycle with values in $U(2)$. But there is a natural embedding $U(2) \subset \operatorname{Spin}^{\mathbb{C}}(4)$ given as

$$
U(2) \longrightarrow \operatorname{Spin}^{\mathbb{C}}(4) \quad: \quad[\lambda, \xi] \longmapsto[\lambda, \lambda, \xi] .
$$

Therefore the $U(2)$-cocycle of $T_{M}$ lifts to a canonical spin ${ }^{\mathbf{C}}$-structure $\left\{\widetilde{\tau}_{\alpha \beta}\right\}$ associated with the almost-complex structure $\omega$.

Concretely, identify the model-fiber of $T_{M}$ with $\mathbb{H}$. If $\left.T_{M}\right|_{U} \approx U \times \mathbb{H}$ and $\left.T_{M}\right|_{U^{\prime}} \approx U^{\prime} \times \mathbb{H}$ are two bundle-charts, write $[\lambda, \xi]: U \cap U^{\prime} \rightarrow \mathbb{S}^{1} \times \mathbb{S}^{3} / \pm 1$ for the associated $U(2)$-transition map, which identifies $(x, v) \in U \times \mathbb{H}$ with $\left(x, v^{\prime}\right) \in U^{\prime} \times \mathbb{H}$ when $v^{\prime}=\lambda(x) v \xi(x)^{-1}$. The associated spin $^{\mathbf{C}}$-structure will have corresponding $\operatorname{Spin}^{\mathbb{C}}(4)$-transition map $[\lambda, \lambda, \xi]: U \cap U^{\prime} \rightarrow \mathbb{S}^{1} \times \mathbb{S}^{3} \times \mathbb{S}^{3} / \pm 1$.

The spinor bundle $\mathcal{W}^{+}$will have model-fiber $\mathbb{H}$ and corresponding $U(2)$-transition map $[\lambda, \lambda]: U \cap U^{\prime} \rightarrow \mathbb{S}^{1} \times \mathbb{S}^{3} / \pm 1$, identifying $\left(x, w_{+}\right)$with $\left(x, w_{+}^{\prime}\right)$ when $w_{+}^{\prime}=$ $\lambda(x) w_{+} \lambda(x)^{-1}$. The spinor bundle $\mathcal{W}^{-}$will have model-fiber $\mathbb{H}$ and corresponding $U(2)$-transition map $[\lambda, \xi]: U \cap U^{\prime} \rightarrow \mathbb{S}^{1} \times \mathbb{S}^{3} / \pm 1$ identifying $\left(x, w_{-}\right)$with $\left(x, w_{-}^{\prime}\right)$ when $w_{-}^{\prime}=\lambda(x) w_{-} \xi(x)^{-1}$. The determinant line bundle $L$ will have model-fiber $\mathbb{C}$ and corresponding $U(1)$-transition function $\lambda^{2}: U \cap U^{\prime} \rightarrow \mathbb{S}^{1}$, identifying $(x, z)$ with $\left(x, z^{\prime}\right)$ when $z^{\prime}=\lambda(x)^{2} z$. (Note that, although the bundles $\mathcal{W}^{ \pm}$have modelfiber $\mathbb{H}$, after gluing them up with their respective cocycles, no global quaternionic structure is preserved, only a complex structure.)

After inspecting the cocycles, immediate consequences are the well-known isomorphisms of Hermitian bundles $\mathcal{W}^{-} \approx T_{M}$ and $L \approx K^{*}$.

Suppose now that $V$ is a 4 -dimensional vector space, endowed with an inner product and with an orientation. Denote by $P S O(V)$ the group of orientation-preserving conformal transformations of $V$ (i.e. real multiples of orthogonal transformations from $S O(V)$ ). Further, denote by $\mathrm{PSO}^{+}(V)$ the group of self-dual conformal transformations, that is, those maps $V \rightarrow V$ which, with respect to orienting orthonormal bases, are represented by matrices of the form

$$
\left[\begin{array}{rrrr}
r \cos \theta & r \sin \theta & & \\
-r \sin \theta & r \cos \theta & & \\
& & r \cos \theta & r \sin \theta \\
& & -r \sin \theta & r \cos \theta
\end{array}\right] .
$$

Note that the zero map $V \rightarrow 0$ is in $\mathrm{PSO}^{+}(V)$, as is the identity $i d: V \rightarrow V$.

Equivalently: Pick any vector-space isomorphism $V \approx \mathbb{H}$ preserving the inner product and the orientation. Then, a self-dual conformal transformation is the same as the map $v \mapsto \xi v$ defined by multiplying on the left with a quaternion $\xi$.

A third description: Any linear $\phi: V \rightarrow V$ induces a map $\phi: \Lambda^{2}(V) \rightarrow \Lambda^{2}(V)$ with $\phi(v \wedge w)=\phi(v) \wedge \phi(w)$. Since $V$ is 4-dimensional, oriented, and has an inner product, we have a splitting $\Lambda^{2}(V)=\Lambda^{+}(V) \oplus \Lambda^{-}(V)$. An automorphism 
$\phi: V \rightarrow V$ is a conformal transformation if and only if it preserves the splitting $\Lambda^{2}=\Lambda^{+} \oplus \Lambda^{-}$. It is a self-dual conformal transformation if and only if in addition it acts trivially on the anti-self-dual part, that is, if $\left.\phi\right|_{\Lambda^{-}(V)}=i d$.

Let $M$ be a closed oriented 4-manifold, endowed with a fixed metric $g$. We can define the bundle $\mathrm{PSO}^{+}\left(T_{M}\right)$ as the subbundle of $\operatorname{Hom}\left(T_{M}, T_{M}\right)$ containing the self-dual conformal transformations of the fibers. It is a vector bundle of rank 4 . As a subbundle of $\operatorname{Hom}\left(T_{M}, T_{M}\right)$, it comes equipped with a fiber-metric induced from $g$, as well as an obvious evaluation map $T_{M} \times P S O^{+}\left(T_{M}\right) \stackrel{\mathrm{ev}}{\longrightarrow} T_{M},(v, \phi) \longmapsto \phi(v)$.

Suppose now that $M$ is endowed with some almost-complex structure $\omega$. Then $T_{M}$ becomes a complex bundle $\left(T_{M}, \omega\right)$. A complex structure is induced on the bundle $\mathrm{PSO}^{+}\left(T_{M}\right)$ simply by $(i \cdot \phi)(v)=i \cdot \phi(v)$. Denote the resulting Hermitian bundle by $\left(P S O^{+}, \omega\right)$.

More, for any $\phi \in P S O^{+}\left(T_{M}\right)$, we can define the pull-back $\phi^{*} \omega$ of the fundamental 2-form $\omega \in \Lambda^{+}\left(T_{M}^{*}\right)$ :

$$
\left(\phi^{*} \omega\right)(v, w)=\omega(\phi v, \phi w) .
$$

Since $\phi$ preserves $\Lambda^{+}$, the pull-back $\phi^{*} \omega$ will be in $\Lambda^{+}\left(T_{M}^{*}\right)$ as well.

Theorem 2.1. Let $M$ be an oriented 4-manifold endowed with a Riemannian metric and a compatible almost-complex structure $\omega$, which induce a spin ${ }^{\mathrm{C}}$-structure with spinor bundles $\mathcal{W}^{+}$and $\mathcal{W}^{-}$.

(A) We have the natural Hermitian bundle isomorphisms

$$
\mathcal{W}^{-} \approx\left(T_{M}, \omega\right) \quad \mathcal{W}^{+} \approx\left(P S O^{+}\left(T_{M}\right), \omega\right) .
$$

(B) The Clifford multiplication $T_{M} \times \mathcal{W}^{+} \stackrel{\bullet}{\longrightarrow} \mathcal{W}^{-}$identifies with the evaluation map $T_{M} \times P_{S O}^{+}\left(T_{M}\right) \stackrel{\mathrm{ev}}{\longrightarrow} T_{M}$ as

$$
v \bullet \phi \equiv \phi(v) .
$$

(C) The squaring map $\sigma: \mathcal{W}^{+} \rightarrow \Lambda^{+}$can be written

$$
\sigma(\phi)=\frac{1}{4} \phi^{*} \omega .
$$

Remark 2.2. Varying the almost-complex structure $\omega$ does not change the underlying real bundles of the spinor bundles, nor the Clifford multiplication map. It only changes the complex structures that are laid on them.

Proof of 2.1(A). We already proved $\mathcal{W}^{-} \approx T_{M}$. To show $\mathcal{W}^{+} \approx P S O^{+}$, we need only uncover the cocycle of the latter. Identify the model-fiber of $T_{M}$ with $\mathbb{H}$. Then a self-dual conformal transformation of $T_{M}$ can be represented fiberwise by left-multiplication with a quaternion. Pick a self-dual conformal transformation $\phi: T_{M} \rightarrow T_{M}$, and consider two bundle-charts $\left.T_{M}\right|_{U} \approx U \times \mathbb{H}$ and $\left.T_{M}\right|_{U^{\prime}} \approx U^{\prime} \times \mathbb{H}$ related by some $U(2)$-transition map $[\lambda, \xi]$. Let $x \in U \cap U^{\prime}$. If in the chart over $U$ the transformation $\phi$ is represented as $v \mapsto h v$ for some $h: U \rightarrow \mathbb{H}$, while in the chart over $U^{\prime}$ it is represented as $v^{\prime} \mapsto h^{\prime} v^{\prime}$ for some $h: U^{\prime} \rightarrow \mathbb{H}$, then, since the coordinate change is $v^{\prime}=\lambda v \xi^{-1}$, we must have $\lambda h v \xi^{-1}=h^{\prime} \lambda v \xi^{-1}$ for all $v$. Therefore $h^{\prime}=\lambda h \lambda^{-1}$. In conclusion, $P S O^{+}\left(T_{M}\right)$ has the same transition functions as $\mathcal{W}^{+}$, and hence these Hermitian complex bundles are isomorphic.

Remark 2.3. The isomorphism $\mathcal{W}^{-} \approx T_{M}$ is well-known, usually written as $\mathcal{W}^{-} \approx$ $\Lambda^{0,1}$. The isomorphism $\mathcal{W}^{+} \approx P S O^{+}$follows immediately from the beginning of [Tau95], Tau00]. For example: The Clifford action of $\omega$ splits $\mathcal{W}^{+}$into \pm 1 eigenbundles as $\mathcal{W}^{+} \approx \mathbb{C} \oplus K^{*}$. But $\Lambda^{+}=\mathbb{R} \omega \oplus K^{*}$. Thus $\mathcal{W}^{+} \approx \mathbb{R} \oplus \Lambda^{+}$. 
Identifying $\Lambda^{+}$with skew-symmetric endomorphisms and writing the trivial component as $\underline{\mathbb{R}}=\mathbb{R} \cdot i d$, we see that $\mathcal{W}^{+} \approx P S O^{+}$as real bundles. The complex structures follow as well.

Proof of [2.1(B). The main task here is to concretely define the Clifford multiplication in such a manner that the identification $v \bullet \phi=\phi(v)$ become obvious.

The action of the structure group $\operatorname{Spin}^{\mathbb{C}}(4)$ on $\mathcal{W}^{+} \oplus \mathcal{W}^{-}$can be extended to an action of the full complexified Clifford algebra $\mathrm{C} \ell(4) \otimes \mathbb{C}$, in a way that respects the inclusion $\operatorname{Spin}^{\mathbb{C}}(4) \subset \mathbb{C} \ell(4)$. Globalizing, we obtain the extension of the action of the principal bundle $\operatorname{Spin}^{\mathbb{C}}\left(T_{M}\right)$ on $\mathcal{W}^{+} \oplus \mathcal{W}^{-}$to an action of the algebra bundle $\mathrm{C} \ell\left(T_{M}\right) \otimes \underline{\mathbb{C}}$.

But $\mathrm{C} \ell(4)$ is isomorphic with the algebra $\mathbb{H}(2)$ of all $2 \times 2$ quaternionic matrices, with $\operatorname{Spin}(4)$ embedded as the group of all matrices

$$
\left[\begin{array}{ll}
\xi_{+} & \\
& \xi_{-}
\end{array}\right]
$$

with $\xi_{ \pm} \in \mathbb{S}^{3}$. The inclusion of $\operatorname{Spin}^{\mathbb{C}}(4)=\operatorname{Spin}(4) \times_{\mathbb{Z}_{2}} \mathbb{S}^{1}$ into $\mathrm{C} \ell(4) \otimes \mathbb{C}$ follows suit.

Identify the model-fiber of $\mathcal{W}^{+} \oplus \mathcal{W}^{-}$with $\mathbb{H} \oplus \mathbb{H}$. We define the Clifford multiplication in local quaternionic coordinates by:

$$
\begin{array}{cccc}
\mathrm{C} \ell\left(T_{M}\right) \otimes \underline{\mathbb{C}} & \times & \mathcal{W}^{+} \oplus \mathcal{W}^{-} \longrightarrow & \mathcal{W}^{+} \oplus \mathcal{W}^{-} \\
{\left[\begin{array}{ll}
a & b \\
c & d
\end{array}\right] \otimes \lambda} & {\left[\begin{array}{ll}
h^{+} & h^{-}
\end{array}\right]} & \lambda \cdot\left[\begin{array}{ll}
h^{+} & h^{-}
\end{array}\right] \cdot\left[\begin{array}{ll}
\bar{a} & \bar{c} \\
\bar{b} & \bar{d}
\end{array}\right]
\end{array}
$$

This local description is compatible with the cocycles of $T_{M}$ and $\mathcal{W}^{ \pm}$, and thus defines a global action of $\mathrm{C} \ell\left(T_{M}\right) \otimes \underline{\mathbb{C}}$ on $\mathcal{W}^{+} \oplus \mathcal{W}^{-}$.

The tangent bundle $T_{M}$ embeds into $\mathrm{C} \ell\left(T_{M}\right)$ via a version of the (quaternionic) Pauli matrices. Namely, for a local identification $\left.T_{M}\right|_{x} \approx \mathbb{H}$, we embed $\left.T_{M}\right|_{x}$ into $\left.\mathrm{C} \ell\left(T_{M}\right)\right|_{x} \approx \mathbb{H}(2)$ through

$$
1 \mapsto\left[\begin{array}{rr}
0 & -1 \\
1 & 0
\end{array}\right], i \mapsto\left[\begin{array}{rr}
0 & -i \\
-i & 0
\end{array}\right], j \mapsto\left[\begin{array}{rr}
0 & -j \\
-j & 0
\end{array}\right], k \mapsto\left[\begin{array}{rr}
0 & -k \\
-k & 0
\end{array}\right] .
$$

(These matrices generate $\mathbb{H}(2)$ and satisfy the Clifford relations $E_{k} \cdot E_{k}=-I d$ and $E_{j} \cdot E_{k}=-E_{k} \cdot E_{j}$. The above are essentially the negatives of the standard Pauli matrices.) In short, the embedding is

$$
v \longmapsto\left[\begin{array}{cc}
\bar{v} & -v
\end{array}\right]
$$

Via this inclusion $T_{M} \subset \mathrm{C} \ell\left(T_{M}\right)$, the tangent vectors will act on spinors as follows:

$$
\begin{array}{ccccccc}
T_{M} \times \mathcal{W}^{+} \longrightarrow & \mathcal{W}^{-} & T_{M} \times \mathcal{W}^{-} & \bullet & \mathcal{W}^{+} \\
v & h^{+} & h^{+} v & v & h^{-} & & -h^{-} \bar{v}
\end{array}
$$

In particular, $v \bullet(v \bullet \phi)=-\|v\|^{2} \phi$, as needed. If we read $T_{M} \times \mathcal{W}^{+} \longrightarrow \mathcal{W}^{-}$ through the isomorphisms $\mathcal{W}^{-} \approx T_{M}$ and $\mathcal{W}^{+} \approx P_{S O}^{+}\left(T_{M}\right)$, we obtain the map $T_{M} \times P_{S O}^{+}\left(T_{M}\right) \longrightarrow T_{M}$ given by $\left(v, h^{+}\right) \longmapsto h^{+} v$. That is exactly the evaluation map read in coordinates. 
Proof of $\left[2.1(\mathrm{C})\right.$. We prove that $\sigma(\phi)=\frac{1}{4} \phi^{*} \omega$. As mentioned before, we use the metric to identify 2-forms with skew-symmetric endomorphisms. Namely, the form $\gamma \in \Gamma\left(\Lambda^{2}\left(T_{M}^{*}\right)\right)$ will correspond to the endomorphism of $T_{M}$ that satisfies

$$
\gamma(v, w)=g(\gamma(v), w)
$$

(We will use the same letter for the 2-form and for the morphism. The context or the specific number of arguments each takes should be enough to distinguish them.) The essential ingredient of the proof is

Lemma 2.4. For any $\beta \in \Lambda^{+}\left(T_{M}\right)$ and $\phi \in \mathcal{W}^{+}$, we have

$$
\beta \bullet \phi=-2 \phi \circ \beta \text {. }
$$

Now, the squaring map $\sigma: \mathcal{W}^{+} \rightarrow \Lambda^{+}\left(T_{M}\right)$ is characterized by

$$
\sigma(\phi) \bullet \phi=-i \frac{\|\phi\|^{2}}{2} \phi \text {. }
$$

Using [2.4, that translates to $\phi \circ \sigma(\phi)=\frac{\|\phi\|^{2}}{4} i \phi$, which can be written as

$$
\phi \circ \sigma(\phi)=\frac{\|\phi\|^{2}}{4} J \circ \phi .
$$

Therefore, thinking of $\phi$ as $\phi: T_{M} \rightarrow T_{M}$, and ignoring the factor $\|\phi\|^{2} / 4$ for a moment, we see that $\sigma(\phi)$ must determine the unique almost-complex structure on $T_{M}$ that will make $\phi:\left(T_{M}, \sigma(\phi)\right) \rightarrow\left(T_{M}, J\right)$ be $\mathbb{C}$-linear. When $\left.\phi\right|_{x}=0$, statement $(\mathrm{C})$ is immediate, while in general we have

$$
\begin{aligned}
\frac{1}{4} \omega(\phi v, \phi w) & =\frac{1}{4} g(J \phi v, \phi w)=\frac{1}{\|\phi\|^{2}} g\left(\frac{\|\phi\|^{2}}{4} J \phi v, \phi w\right) \\
& =\frac{1}{\|\phi\|^{2}} g(\phi \sigma(\phi) v, \phi w)=g(\sigma(\phi) v, w) \\
& =\sigma(\phi)(v, w)
\end{aligned}
$$

(where we used that $\phi$ is conformal, and hence $g(\phi x, \phi y)=\|\phi\|^{2} g(x, y)$ ). Thus $\frac{1}{4} \phi^{*} \omega=\sigma(\phi)$.

Remark 2.5. Statement 2.1(C) interprets the square $\sigma(\phi)$ as a (weighted) pull-back of the almost-complex structure $\omega$ through $\phi: T_{M} \rightarrow\left(T_{M}, \omega\right)$. On the one hand, it is worth quoting the following statement:

Let $\alpha$ be a self-dual 2-form, and assume that $H^{2}(M \backslash\{$ zeros of $\alpha\} ; \mathbb{Z})$ has no 2 -torsion. There is a self-dual spinor field $\phi \in \Gamma\left(\mathcal{W}^{+}\right)$such that $\alpha=\phi^{*} \omega$ if and only if $c_{1}\left(\left.\alpha\right|_{\text {off zeros }}\right)=\left.c_{1}(\omega)\right|_{\text {off zeros. }}$. Sco02]

On the other hand, if we fix a suitable self-dual 2-form $\alpha$, we can ask how unique is a spinor field $\phi$ such that $\alpha=\phi^{*} \omega$. Such a $\phi: T_{M} \rightarrow T_{M}$ has the homothety ratio prescribed from $\|\phi\|^{2}=2 \sqrt{2}\left\|\phi^{*} \omega\right\|$, and must map the complex planes of $\alpha$ onto the complex planes of $\omega$. Nonetheless, it has the freedom of rotating those planes. Concretely, if $\left.\phi^{*} \omega\right|_{x}=\left.\psi^{*} \omega\right|_{x}$, then $\left.\phi\right|_{x}=\left.e^{i \theta} \psi\right|_{x}$ for some angle $\theta$. This angular freedom can be factored out using the gauge group $\mathcal{G}=\left\{f: M \rightarrow \mathbb{S}^{1}\right\}$.

Proof of Lemma[2.4 We show that $\beta \bullet \phi=2 \phi \circ \beta$. Through the Clifford action of 2 -forms on $\mathcal{W}^{+} \oplus \mathcal{W}^{-}$, every 2 -form acts on a self-dual spinor only through its self-dual part (the anti-self-dual part acts trivially). For example, if $\left.\phi \in \mathcal{W}^{+}\right|_{x}$ and $\left\{a_{1}, a_{2}, a_{3}, a_{4}\right\}$ is any orienting orthonormal basis in $\left.T_{M}\right|_{x}$, we have

$$
\left(a_{1} \wedge a_{2}\right) \bullet \phi=\left(a_{3} \wedge a_{4}\right) \bullet \phi=\frac{1}{2}\left(a_{1} \wedge a_{2}+a_{3} \wedge a_{4}\right) \bullet \phi .
$$


Through the isomorphism $\mathrm{C} \ell\left(T_{M}\right) \approx \Lambda\left(T_{M}\right)$, the algebra multiplication in $\mathrm{C} \ell\left(T_{M}\right)$ can be expressed as $\left.v \cdot \gamma=v \wedge \gamma-v\right\lrcorner \gamma$, for $v \in T_{M}$ and any $\gamma \in \mathrm{C} \ell\left(T_{M}\right)$. Here $\lrcorner$ is the interior product $v\lrcorner \gamma=\gamma(v, \cdot, \ldots, \cdot)$ (identify $\Lambda\left(T_{M}\right)$ and $\Lambda\left(T_{M}^{*}\right)$ ). If $v$ and $w$ are orthogonal, then $v\lrcorner w=0$, and hence $v \cdot w=v \wedge w$. In particular, $\left(a_{1} \wedge a_{2}\right) \bullet \phi=\left(a_{1} \cdot a_{2}\right) \bullet \phi$ and $\left(a_{3} \wedge a_{4}\right) \bullet \phi=\left(a_{3} \cdot a_{4}\right) \bullet \phi$.

Therefore we have $\psi=\frac{1}{2}\left(a_{1} \wedge a_{2}+a_{3} \wedge a_{4}\right) \bullet \phi$ if and only if $\psi=\left(a_{1} \cdot a_{2}\right) \bullet \phi$ and $\psi=\left(a_{3} \cdot a_{4}\right) \bullet \phi$. Since $a_{k} \cdot a_{k}=-1$, that is the same as $a_{1} \bullet \psi=-a_{2} \bullet \phi$ and $a_{3} \bullet \psi=-a_{4} \bullet \phi$. Using the identification $\mathcal{W}^{+} \approx P S O^{+}\left(T_{M}\right)$, that becomes

$$
\psi=\frac{1}{2}\left(a_{1} \wedge a_{2}+a_{3} \wedge a_{4}\right) \bullet \phi \quad \Longleftrightarrow \quad \psi\left(a_{1}\right)=-\phi\left(a_{2}\right) \quad \& \quad \psi\left(a_{3}\right)=-\phi\left(a_{4}\right) .
$$

The element $\alpha=a_{1} \wedge a_{2}+a_{3} \wedge a_{4}$ of $\left.\Lambda^{+}\right|_{x}$ corresponds to the rotation $\alpha:\left.T_{M}\right|_{x} \rightarrow$ $\left.T_{M}\right|_{x}$ acting by $a_{1} \mapsto a_{2}, a_{2} \mapsto-a_{1}$, and $a_{3} \mapsto a_{4}, a_{4} \mapsto-a_{3}$. The composition $\phi \circ \alpha$ will still be self-dual, and will act by $(\phi \circ \alpha)\left(a_{1}\right)=\phi\left(a_{2}\right)$ and $(\phi \circ \alpha)\left(a_{3}\right)=\phi\left(a_{4}\right)$. Comparing with $\psi$, we conclude that

$$
\alpha \bullet \phi=-2 \phi \circ \alpha .
$$

To get Lemma 2.4 we need only remark that for any $\left.\beta \in \Lambda^{+}\right|_{x}$, there is always a suitable basis $\left\{b_{1}, b_{2}, b_{3}, b_{4}\right\}$ in $\left.T_{M}\right|_{x}$ so that $\beta=r\left(b_{1} \wedge b_{2}+b_{3} \wedge b_{4}\right)$, with $r=\frac{1}{\sqrt{2}}\|\beta\|$, and thus the above applies.

\section{Geometry}

Using the language of Theorem 2.1 we view a self-dual spinor field as a bundle morphism $\phi:\left(T_{M}, g, \nabla\right) \rightarrow\left(T_{M}, g, \omega\right)$. The connections $\widetilde{\nabla}$ on $\mathcal{W}^{-}$are seen as connections on the target of such $\phi$.

Choose any unitary connection $A$ on $K^{*}$. This $A$ can be combined with the LeviCività connection $\nabla$ to induce unitary connections $\bar{\nabla}^{A}$ on $\mathcal{W}^{+}$and $\widetilde{\nabla}^{A}$ on $\mathcal{W}^{-}$. That is done by lifting $A$ through the map $\operatorname{Spin}^{\mathbb{C}}(4) \rightarrow U(1)$, combining with the lift of $\nabla$ through $\operatorname{Spin}^{\mathbb{C}}(4) \rightarrow S O(4)$, and then projecting the combination through the two maps $\operatorname{Spin}^{\mathbb{C}}(4) \rightarrow U(2)$. (For details, see [LM89, [Mor96], or the proof of Lemma 3.1 at the end of this paper.) We call such connections on $\mathcal{W}^{ \pm}$spinorial connections.

Viewing a spinorial connection $\widetilde{\nabla}$ on $\mathcal{W}^{-}$as a connection on $T_{M}\left(\operatorname{via} \mathcal{W}^{-} \approx T_{M}\right)$, it is obvious that $\widetilde{\nabla}$ is $\mathbb{C}$-linear for $\omega$ and $g$-metric. In fact:

Lemma 3.1. Let $D$ be any connection on $T_{M}$. There is a connection $A$ on $K^{*}$ such that $D=\widetilde{\nabla}^{A}$ (via the identification $T_{M} \approx \mathcal{W}^{-}$) if and only if $D$ is g-metric, $\mathbb{C}$-linear for $\omega$, and the induced connection $\left.D\right|_{\Lambda^{-}}$on $\Lambda^{-}\left(T_{M}^{*}\right)$ coincides with the one induced by the Levi-Cività connection $\nabla$.

Remark 3.2. Given $D$ as above, one can find the suitable $A$ as follows: The fact that $D$ is $\mathbb{C}$-linear for $\omega$ can also be written as $\left.D\right|_{\Lambda^{+}} \omega=0$. Since $\Lambda^{+}=\mathbb{R} \omega \oplus K^{*}$, that implies that $\left.D\right|_{\Lambda^{+}}=\partial \oplus A$ for some unitary connection $A$ on $K^{*}$ (where $\partial$ denotes the trivial connection on the trivialized bundle $\mathbb{R} \omega$ ). If the conditions from 3.1 are met, then $D=\widetilde{\nabla}^{A}$.

Remark 3.3. The fact that connections $D$ with $\left.D\right|_{\Lambda^{-}}=\left.\nabla\right|_{\Lambda^{-}}$play such an important rôle in spin-geometry could be justified in the language of [2.1 as follows: A bundle morphism $\phi: T_{M} \rightarrow T_{M}$ is a self-dual spinor field if and only if the induced map on $\Lambda^{2}$ preserves the splitting $\Lambda^{2}=\Lambda^{+} \oplus \Lambda^{-}$and acts on $\Lambda^{-}$as the identity. It is 
thus natural that the most natural connections on the target of $\phi:\left(T_{M}, \nabla\right) \rightarrow T_{M}$ coincide with $\nabla$ on $\Lambda^{-}$.

Lemma 3.1 suggests a non-standard approach: Instead of choosing $A$ and building $\widetilde{\nabla}^{A}$ and $\bar{\nabla}^{A}$, one could start with a connection $\widetilde{\nabla}$ on $T_{M}$ which is $g$-metric, $\mathbb{C}$-linear, and has $\left.\widetilde{\nabla}\right|_{\Lambda^{-}}=\left.\nabla\right|_{\Lambda^{-}}$. We know that $\widetilde{\nabla}=\widetilde{\nabla}^{A}$ for some $A$, but we do not determine $A$. Instead, seeing spinor fields as $\phi:\left(T_{M}, \nabla\right) \rightarrow\left(T_{M}, \widetilde{\nabla}\right)$ and using (11) in the form

$$
(\bar{\nabla} \phi) v=\widetilde{\nabla}(\phi v)-\phi(\nabla v)
$$

we can define a connection $\bar{\nabla}$ on the whole $\operatorname{Hom}\left(T_{M}, T_{M}\right)$. Because of 3.1 we know that this $\bar{\nabla}$ must preserve the subbundle $\mathcal{W}^{+} \subset \operatorname{Hom}\left(T_{M}, T_{M}\right)$. The restriction of $\bar{\nabla}$ to $\mathcal{W}^{+}$is then exactly $\bar{\nabla}^{A}$.

The Hermitian identification $\mathcal{W}^{-} \approx\left(T_{M}, g, \omega\right)$ further tempts one to consider connections $\tilde{\nabla}$ on $\mathcal{W}^{-}$that are merely $g$-metric and $\mathbb{C}$-linear for $\omega$. We call them admissible connections on $\mathcal{W}^{-}$.

Admissible connections no longer correspond to a connection $\bar{\nabla}$ on $\mathcal{W}^{+}$. Nonetheless, using (5), we can still define a connection $\bar{\nabla}$ on $\operatorname{Hom}\left(T_{M}, T_{M}\right)$, but it will no longer preserve the subbundle $\mathcal{W}^{+}$. The meaning of $\bar{\nabla}$ (be it spinorial or not), as read from (5), is to compare the connections $\nabla$ and $\widetilde{\nabla}$ through $\phi:\left(T_{M}, g, \nabla\right) \rightarrow$ $\left(T_{M}, g, \omega, \widetilde{\nabla}\right)$. For easier manipulation, we introduce the notation

$$
\left(\mathcal{B}^{\widetilde{\nabla}} \phi\right)_{X} Y=\left(\bar{\nabla}_{X} \phi\right)(Y)=\widetilde{\nabla}_{X}(\phi Y)-\phi\left(\nabla_{X} Y\right)
$$

which can be thought of as the analogue of a "second fundamental form" for $\phi$ : $T_{M} \rightarrow T_{M}$.

We already encountered the pull-back $\phi^{*} \omega=4 \sigma(\phi)$ of the fundamental form. We can also pull-back the metric $g$ to $\phi^{*} g$, given by $\left(\phi^{*} g\right)(v, w)=g(\phi v, \phi w)$. Since $\phi$ is conformal, it is simply $\phi^{*} g=\|\phi\|^{2} g$. We can as well pull-back the unitary connection $\widetilde{\nabla}$ to $\phi^{*} \widetilde{\nabla}$, defined by $\left(\phi^{*} \widetilde{\nabla}\right)_{X} Y=\phi^{-1} \widetilde{\nabla}_{X}(\phi Y)$, where $\phi^{-1}$ is the inverse of $\phi$ (defined only off the zeros of $\phi$ ). Off the zeros of $\phi$, the comparison form $\mathcal{B}^{\tilde{\nabla}_{\phi}}$ can then be written $\left(\mathcal{B}^{\tilde{\nabla}} \phi\right)_{X} Y=\phi\left(\left(\phi^{*} \widetilde{\nabla}\right)_{X} Y-\nabla_{X} Y\right)$.

The zeros of $\phi$ create singularities, but, nonetheless, since $g, \omega$, and $\widetilde{\nabla}$ were compatible, so will their pull-backs. Namely: the form $\phi^{*} \omega$ is self-dual and has constant length $\sqrt{2}$ for the (singular) metric $\phi^{*} g$, and thus corresponds to a (singular) almost-complex structure; the (singular) connection $\phi^{*} \widetilde{\nabla}$ is Hermitian, i.e. it is $\phi^{*} g$-metric and $\mathbb{C}$-linear for $\phi^{*} \omega$.

Remark 3.4. The zeros of spinors are hard to control. Even in the case of harmonic spinors (spinor fields $\phi$ for which there is an $A$ such that $\mathcal{D}^{A} \phi=0$ ), the zero-set is a countable 2-rectifiable set, and thus has Hausdorff dimension as high as 2 (see [Bär97]).

In general, the connection $\phi^{*} \widetilde{\nabla}$ will have torsion. But if, for example, $\mathcal{B}^{\widetilde{\nabla}} \phi=0$, then $\bar{\nabla} \phi=0$, and so $\phi$ has constant length. If further $\phi$ is non-trivial, then $\phi^{*} \widetilde{\nabla}$ is well-defined on all $M$, and we have $\phi^{*} \widetilde{\nabla}=\nabla$. In this case $\left(M, \phi^{*} g, \phi^{*} \omega\right)$ is a Kähler manifold. This suggests that the comparison form $\mathcal{B} \phi$ has control over the geometry of the deformed structure $\phi^{*} \omega$.

If $V$ is a vector space endowed with an inner product $q$, we have the standard splitting $V \otimes V=\Lambda^{2}(V) \oplus \mathrm{S}_{0}^{2}(V) \oplus \mathbb{R} q$. The comparison form $\mathcal{B}^{\tilde{\nabla}} \phi$ is tensorial - 
a section in $T_{M}^{*} \otimes T_{M}^{*} \otimes T_{M}$. Applying the above splitting on the $T_{M}^{*} \otimes T_{M}^{*}$-factor, we get the splitting

$$
\mathcal{B}^{\tilde{\nabla}} \phi=\operatorname{Alt} \mathcal{B}^{\tilde{\nabla}} \phi+\operatorname{Sym}_{0} \mathcal{B}^{\tilde{\nabla}} \phi+g \otimes \frac{1}{4} \operatorname{tr}\left(\mathcal{B}^{\tilde{\nabla}} \phi\right)
$$

which breaks $\mathcal{B}^{\tilde{\nabla}} \phi$ into its skew-symmetric, traceless-symmetric, and trace parts. Concretely, these are defined as

$$
\begin{aligned}
& \left(\operatorname{Alt} \mathcal{B}^{\widetilde{\nabla}} \phi\right)_{X} Y=\frac{1}{2}\left(\left(\mathcal{B}^{\widetilde{\nabla}} \phi\right)_{X} Y-\left(\mathcal{B}^{\widetilde{\nabla}} \phi\right)_{Y} X\right), \\
& \left(\operatorname{Sym} \mathcal{B}^{\widetilde{\nabla}} \phi\right)_{X} Y=\frac{1}{2}\left(\left(\mathcal{B}^{\widetilde{\nabla}} \phi\right)_{X} Y+\left(\mathcal{B}^{\widetilde{\nabla}} \phi\right)_{Y} X\right), \\
& \operatorname{Sym}_{0} \mathcal{B}^{\widetilde{\nabla}} \phi=\operatorname{Sym} \mathcal{B}^{\widetilde{\nabla}} \phi-g \otimes \frac{1}{4} \operatorname{tr}\left(\mathcal{B}^{\widetilde{\nabla}} \phi\right), \\
& \operatorname{tr} \mathcal{B}^{\widetilde{\nabla}} \phi=\left\langle\mathcal{B}^{\widetilde{\nabla}} \phi, g\right\rangle=\sum\left(\mathcal{B}^{\widetilde{\nabla}} \phi\right)_{e_{k}} e_{k}
\end{aligned}
$$

where $\left\{e_{1}, e_{2}, e_{3}, e_{4}\right\}$ is any $g$-orthonormal basis in $\left.T_{M}\right|_{x}$. When $\widetilde{\nabla}=\widetilde{\nabla}^{A}$ is spinorial, we denote $\mathcal{B}^{\tilde{\nabla}}$ by $\mathcal{B}^{A}$, as expected. Notice that in that case $\operatorname{tr}\left(\mathcal{B}^{A} \phi\right)$ coincides with $\mathcal{D}^{A} \phi$, the Dirac operator.

The skew-symmetric component Alt $\mathcal{B} \phi$ is easy to interpret. Indeed, off the zeros of $\phi$, the following calculation holds:

$$
\begin{aligned}
& \left(\operatorname{Alt} \mathcal{B}^{\tilde{\nabla}}\right)_{X} Y=\frac{1}{2}\left(\widetilde{\nabla}_{X}(\phi Y)-\widetilde{\nabla}_{Y}(\phi X)-\phi\left(\nabla_{X} Y\right)+\phi\left(\nabla_{Y} X\right)\right) \\
& =\frac{1}{2}\left(\phi \phi^{-1} \widetilde{\nabla}_{X} \phi Y-\phi \phi^{-1} \widetilde{\nabla}_{Y} \phi X-\phi[X, Y]-\phi\left(\nabla_{X} Y-\nabla_{Y} X-[X, Y]\right)\right) \\
& =\frac{1}{2} \phi\left(\operatorname{Tor}^{\phi^{*}} \widetilde{\nabla}(X, Y)-\operatorname{Tor}^{\nabla}(X, Y)\right)
\end{aligned}
$$

where $\operatorname{Tor}^{D}(X, Y)=D_{X} Y-D_{Y} X-[X, Y]$ denotes the torsion of a connection $D$. Thus Alt $\mathcal{B}^{\tilde{\nabla}} \phi$ is the torsion-comparing component of $\mathcal{B}^{\widetilde{\nabla}} \phi$. Since $\nabla$ has no torsion, we simply have

$$
\left(\operatorname{Alt} \mathcal{B}^{\tilde{\nabla}} \phi\right)_{X} Y=\frac{1}{2} \phi\left(\operatorname{Tor}^{\phi^{*}} \tilde{\nabla}(X, Y)\right) .
$$

We are now ready to prove Theorem 1.2 stated as:

The equality $\alpha=\phi^{*} \omega$ establishes a bijection between: the set of all Kähler forms $\alpha$ with $c_{1}(\alpha)=c_{1}(\omega)$ and compatible with a metric conformal to $g$; and the set of all gauge classes of pairs $(\phi, \widetilde{\nabla})$ with $\widetilde{\nabla}$ admissible, $\phi$ nowhere-zero, and with Alt $\mathcal{B}^{\tilde{\nabla}} \phi=0$.

Proof of Theorem 1.2. We have Alt $\mathcal{B}^{\tilde{\nabla}} \phi=0$ if and only if $\phi^{*} \widetilde{\nabla}$ is torsion-free. But in that case $\phi^{*} \widetilde{\nabla}$ is the Levi-Cività connection of $\phi^{*} g$, and since it is also $\mathbb{C}$-linear for $\phi^{*} \omega$, we conclude that the form $\phi^{*} \omega$ is Kähler for the metric $\phi^{*} g$.

Conversely, assume $M$ admits a Kähler form $\alpha$ for a metric $g^{\prime}$ conformal to $g$, and has the same Chern class as $\omega$. The latter implies (by Remark 2.5) that there is a nowhere-zero spinor field $\phi$ such that $\phi^{*} \omega=\alpha$. Since $\alpha$ has length $\sqrt{2}$ for $g^{\prime}$, we deduce that we must also have $\phi^{*} g=g^{\prime}$. If $\nabla^{\prime}$ denotes the Levi-Cività connection of $g^{\prime}$, then $\nabla^{\prime} \alpha=0$. Define $\widetilde{\nabla}=\phi_{*} \nabla^{\prime}$, where $\left(\phi_{*} \nabla^{\prime}\right)_{X} Y=\phi \nabla_{X}^{\prime}\left(\phi^{-1} Y\right)$. Then $\widetilde{\nabla}$ is an admissible connection on $T_{M}$ (it is $g$-metric and $\mathbb{C}$-linear for $\omega$ ), and obviously has $\phi^{*} \widetilde{\nabla}=\nabla^{\prime}$. The latter being torsion-free, we must have Alt $\mathcal{B}^{\tilde{\nabla}} \phi=0$. The gauge-invariance part follows easily.

Remark 3.5. Unless $\phi$ has constant length, or, equivalently, unless the metric $g^{\prime}=$ $\phi^{*} g$ is a scalar multiple of $g$ (as in [1.1), the connection $\widetilde{\nabla}$ is not spinorial. 
Combining the bijections from Theorem 1.2 and Proposition 1.1 yields a coarse constraint that Alt $\mathcal{B}$ imposes on the whole $\mathcal{B}$ :

Lemma 3.6. Assume that, for a spinor field $\phi$, we have $\operatorname{Alt} \mathcal{B}^{A} \phi=0$. If $\phi$ is non-trivial and has constant length, then $\mathcal{B}^{A} \phi=0$.

We now restrict our attention to the standard spinorial connections $\bar{\nabla}^{A}$ and $\widetilde{\nabla}^{A}$. Varying these means varying $A$. Denote by $\mathcal{C}$ onn $\left(K^{*}\right)$ the set of all unitary connections on $K^{*}$. Any two connections from $\mathcal{C}$ onn $\left(K^{*}\right)$ differ by a global imaginary 1-form. When $A$ varies in $\operatorname{Conn}\left(K^{*}\right)$, that is when $A$ changes to $A+2 i \theta$ for some $\theta \in \Gamma\left(T_{M}^{*}\right)$, then $\widetilde{\nabla}^{A}$ changes to $\widetilde{\nabla}^{A+2 i \theta}=\widetilde{\nabla}^{A}+i \theta$. The associated tensors change as follows:

$$
\begin{aligned}
& \left(\mathcal{B}^{A+2 i \theta} \phi\right)_{X} Y=\left(\mathcal{B}^{A} \phi\right)_{X} Y+i \theta(X) \phi(Y) \\
& \left(\operatorname{Alt} \mathcal{B}^{A+2 i \theta} \phi\right)_{X} Y=\left(\operatorname{Alt} \mathcal{B}^{A} \phi\right)_{X} Y+\frac{i}{2} \theta(X) \phi(Y)-\frac{i}{2} \theta(Y) \phi(X), \\
& \left(\operatorname{Sym} \mathcal{B}^{A+2 i \theta} \phi\right)_{X} Y=\left(\operatorname{Sym} \mathcal{B}^{A} \phi\right)_{X} Y+\frac{i}{2} \theta(X) \phi(Y)+\frac{i}{2} \theta(Y) \phi(X), \\
& \mathcal{D}^{A+2 i \theta} \phi=\mathcal{D}^{A} \phi+\sum i \theta\left(e_{k}\right) \phi\left(e_{k}\right)=\mathcal{D}^{A} \phi+i \theta \bullet \phi
\end{aligned}
$$

A simple consequence of these formulae is:

If, for two connections $A$ and $A^{\prime}$, we have $\mathcal{B}^{A} \phi=\mathcal{B}^{A^{\prime}} \phi$, or $\operatorname{Alt} \mathcal{B}^{A} \phi=\operatorname{Alt} \mathcal{B}^{A^{\prime}} \phi$, or $\operatorname{Sym} \mathcal{B}^{A} \phi=\operatorname{Sym} \mathcal{B}^{A^{\prime}} \phi$, or $\mathcal{D}^{A} \phi=\mathcal{D}^{A^{\prime}} \phi$, then we must have $A=A^{\prime}$ on the support of $\phi$.

The same formulae also yield:

Theorem 3.7. Let $\left(\mathcal{B}^{A} \phi\right)_{X} Y=Y \bullet \bar{\nabla}_{X}^{A} \phi$ and vary $A$. For every nowhere-zero spinor field $\phi$, there are a unique connection $A_{0}$ on $K^{*}$ and a unique 1-form $\xi \in$ $\Gamma\left(T_{M}^{*}\right)$ such that

$$
\begin{array}{ll}
A=A_{0} & \text { is the unique minimum point of } \mathcal{B}^{A} \phi, \\
A=A_{0}-3 i \xi & \text { is the unique minimum point of } \operatorname{Sym} \mathcal{B}^{A} \phi, \\
A=A_{0}+5 i \xi & \text { is the unique minimum point of } \operatorname{Alt} \mathcal{B}^{A} \phi, \\
A=A_{0}+15 i \xi & \text { is the unique vanishing point of } \mathcal{D}^{A} \phi=\operatorname{tr}\left(\mathcal{B}^{A} \phi\right) .
\end{array}
$$

All these minimizing connections lie on a same affine line in $\mathcal{C}$ onn $\left(K^{*}\right)$. If any two of them happen to coincide, then all of them must coincide.

Theorem 3.8. The formula for $\xi$ above is

$$
\xi=-\frac{4}{15\|\phi\|^{2}} d^{*} \sigma(\phi) \text {. }
$$

Note that $d^{*} \sigma(\phi)=* d \sigma(\phi)$, since $\sigma(\phi)$ is self-dual.

The minima above are determined pointwise. For a fixed $\phi$, let $\mathcal{T}$ denote any one of $\mathcal{B} \phi, \operatorname{Alt} \mathcal{B} \phi, \operatorname{Sym} \mathcal{B} \phi, \operatorname{Sym}_{0} \mathcal{B} \phi$, or $g \otimes \frac{1}{4} \operatorname{tr} \mathcal{B} \phi$. Then the form $\left.\mathcal{T}^{A}\right|_{x}$ is an element of $\left.T_{M}^{*} \otimes T_{M}^{*} \otimes T_{M}\right|_{x}$. The latter has a natural real inner product induced from $g$, given by $\left\langle T_{1}, T_{2}\right\rangle=\sum_{j, k=1}^{4}\left\langle T_{1}\left(e_{j}, e_{k}\right), T_{2}\left(e_{j}, e_{k}\right)\right\rangle$ for any $g$-orthonormal basis $\left\{e_{1}, e_{2}, e_{3}, e_{4}\right\}$ in $\left.T_{M}\right|_{x}$. (All inner products that appear in this paper are real-valued.)

On the other hand, the space $\operatorname{Conn}\left(K^{*}\right)$ of all connections $A$ on $K^{*}$ is affine, with model space $i \Gamma\left(T_{M}^{*}\right)$. The map $\left.A \longmapsto \mathcal{T}^{A}\right|_{x}$ is an affine map $\operatorname{Conn}\left(K^{*}\right) \rightarrow$ $\left.T_{M}^{*} \otimes T_{M}^{*} \otimes T_{M}\right|_{x}$. The image of $\mathcal{C}$ onn $\left(K^{*}\right)$ through this map is an affine subspace $\mathcal{C}_{x}$ in $\left.T_{M}^{*} \otimes T_{M}^{*} \otimes T_{M}\right|_{x}$. Thus, the minimum of the map is unique, and is exactly the 


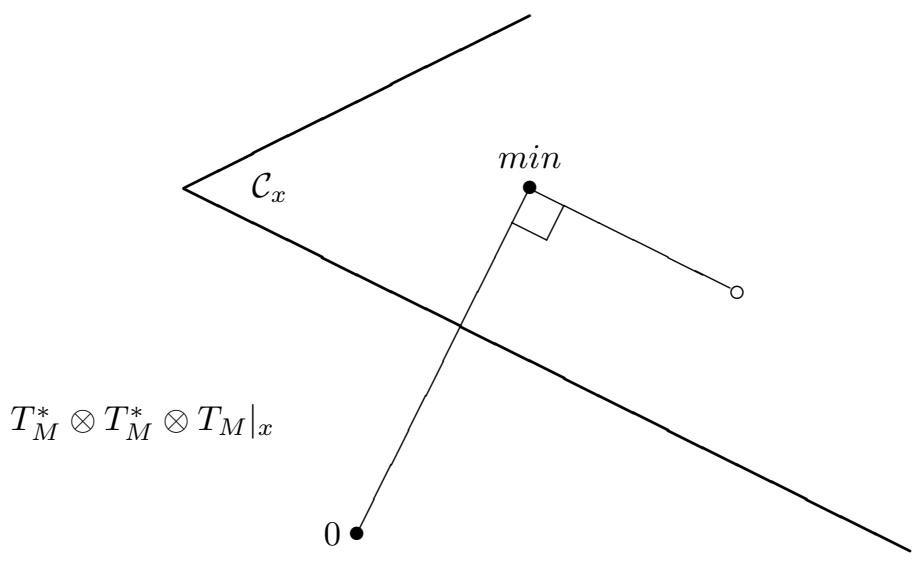

Figure 1. Finding the minimum of $\left.A \longmapsto \mathcal{T}^{A}\right|_{x}$.

point of $\mathcal{C}_{x}$ that is closest to the origin of $\left.T_{M}^{*} \otimes T_{M}^{*} \otimes T_{M}\right|_{x}$. It is the point where the perpendicular from the origin hits $\mathcal{C}_{x}$; see Figure 1 Therefore the map $\left.A \longmapsto \mathcal{T}^{A}\right|_{x}$ attains an absolute minimum at $A_{0}$ if and only if, for any other connection $A$, we have $\left\langle\mathcal{T}^{A_{0}} \phi, \mathcal{T}^{A_{0}} \phi-\left.\mathcal{T}^{A} \phi\right|_{x}=0\right.$. Or, by decoding the inner product, if we have

$$
\left.\sum_{j, k=1}^{4}\left\langle\left(\mathcal{T}^{A_{0}} \phi\right)_{e_{j}} e_{k},\left(\mathcal{T}^{A_{0}} \phi\right)_{e_{j}} e_{k}-\left(\mathcal{T}^{A} \phi\right)_{e_{j}} e_{k}\right\rangle\right|_{x}=0
$$

for some $g$-orthonormal basis $\left\{e_{1}, e_{2}, e_{3}, e_{4}\right\}$ in $\left.T_{M}\right|_{x}$.

Applying the above and using the formulae (6) yields, after some elementary computations,

Lemma 3.9. Let $\left(B^{A} \phi\right)_{X} Y=Y \bullet \bar{\nabla}_{X}^{A} \phi$ and vary $A$. Then:

(A) The comparison form $\left.\mathcal{B}^{A_{0}} \phi\right|_{x}$ is minimal in $\left.T_{M}^{*} \otimes T_{M}^{*} \otimes T_{M}\right|_{x}$ if and only if, for all $\left.X \in T_{M}\right|_{x}$, we have

$$
\left\langle\bar{\nabla}_{X}^{A_{0}} \phi,\left.i \phi\right|_{\left.\right|_{x}}=0\right.
$$

(B) The form $\left.\operatorname{Sym} \mathcal{B}^{A_{0}} \phi\right|_{x}$ is minimal in $\left.T_{M}^{*} \otimes T_{M}^{*} \otimes T_{M}\right|_{x}$ if and only if, for all $\left.X \in T_{M}\right|_{x}$, we have

$$
\left.\left\langle\mathcal{D}^{A_{0}} \phi, i \phi(X)\right\rangle\right|_{x}=\left.6\left\langle\bar{\nabla}_{X}^{A_{0}} \phi, i \phi\right\rangle\right|_{x} .
$$

(C) The form Alt $\left.\mathcal{B}^{A_{0}} \phi\right|_{x}$ is minimal in $\left.T_{M}^{*} \otimes T_{M}^{*} \otimes T_{M}\right|_{x}$ if and only if, for all $\left.X \in T_{M}\right|_{x}$, we have

$$
\left.\left\langle\mathcal{D}^{A_{0}} \phi, i \phi(X)\right\rangle\right|_{x}=-\left.2\left\langle\bar{\nabla}_{X}^{A_{0}} \phi, i \phi\right\rangle\right|_{x} .
$$

(D) Finally, $g \otimes \frac{1}{4} \operatorname{tr}\left(\left.\mathcal{B}^{A_{0}} \phi\right|_{x}\right)=\left.g \otimes \frac{1}{4} \mathcal{D}^{A_{0}} \phi\right|_{x}$ is minimal in $\left.T_{M}^{*} \otimes T_{M}^{*} \otimes T_{M}\right|_{x}$ if and only if

$$
\left.\mathcal{D}^{A_{0}} \phi\right|_{x}=0
$$

Of course, 3.9(D) is utterly trivial. It is included only for completeness.

Remark 3.10. All the above pointwise minima can be realized globally on $M$ if $\phi$ is nowhere-zero. For example, for minimizing $\mathcal{B} \phi$, start with a random connection $A$ and define $\theta_{0}(X)=-\frac{1}{\|\phi\|^{2}}\left\langle\bar{\nabla}_{X}^{A} \phi, i \phi\right\rangle$. Then $A_{0}=A+2 i \theta_{0}$ is the unique 
connection that minimizes $\mathcal{B} \phi$. If $\phi$ has zeros, though, then the globally minimizing connection $A$ might explode at the zeros. (Similarly for $\operatorname{Sym} \mathcal{B} \phi$, Alt $\mathcal{B} \phi$ and $\mathcal{D} \phi$.) Nonetheless, for simplicity from now on we will talk only of global minimizing connections, implicitly restricting away from the possible singularities.

Proof of Corollary 1.3. Lemma 3.9. (A) gives meaning to the condition $\langle\bar{\nabla} \phi, i \phi\rangle=0$ from Proposition [1.1(B): it insures the minimality of $\mathcal{B}^{A} \phi$. Together with the condition $\mathcal{D}^{A} \phi=0$, it implies that all the minimizing connections from Theorem 3.7 coincide (see also 3.8). We could thus rephrase Proposition 1.1(B) as:

The form $\phi^{*} \omega$ is symplectic if and only if there is a connection $A$ that simultaneously minimizes $\mathcal{B}^{A} \phi, \operatorname{Alt}^{A} \mathcal{B} \phi, \operatorname{Sym}^{A} \mathcal{B} \phi$, and $\mathcal{D}^{A} \phi$.

In particular, it is equivalent to:

The form $\phi^{*} \omega$ is symplectic if and only if there is a connection $A$ such that $\mathcal{B}^{A} \phi$ and $\operatorname{Alt} \mathcal{B}^{A} \phi$ are both minimal.

That can be stated as follows:

The form $\phi^{*} \omega$ is symplectic if and only if there is a connection $\widetilde{\nabla}^{A}$ on $T_{M}=$ $\mathcal{W}^{-}$which, through $\phi$, is simultaneously a closest match to $\nabla$ and has the torsion minimized.

In the special case when $\phi=i d: T_{M} \rightarrow T_{M}$, we read:

The manifold $(M, g, \omega)$ is almost-Kähler if and only if there is a $\mathbb{C}$-linear $g$ metric connection $\widetilde{\nabla}$ on $T_{M}$ with $\left.\widetilde{\nabla}\right|_{\Lambda^{-}}=\left.\nabla\right|_{\Lambda^{-}}$, which is simultaneously closest to $\nabla$ and has minimal torsion.

This last statement is exactly Corollary 1.3

Proof of Theorem 3.7. We relate the minimizing connections through the conditions from Lemma 3.9. Assume first that $A$ is the minimizing connection for $\mathcal{B} \phi$, i.e. assume that, for all $X$,

$$
\left\langle\bar{\nabla}_{X}^{A} \phi, i \phi\right\rangle=0
$$

Assume also that $A+2 i \theta$ is the minimizing connection for Alt $\mathcal{B} \phi$, or that, for all $X$,

$$
2\left\langle\bar{\nabla}_{X}^{A+2 i \theta} \phi, i \phi\right\rangle+\left\langle\mathcal{D}^{A+2 i \theta} \phi, i \phi(X)\right\rangle=0 .
$$

Since $\bar{\nabla}_{X}^{A+2 i \theta} \phi=\bar{\nabla}_{X}^{A} \phi+i \theta(X) \phi$, we get

$$
2\|\phi\|^{2} \theta(X)+\left\langle\mathcal{D}^{A+2 i \theta} \phi, i \phi(X)\right\rangle=0 .
$$

Identifying $T_{M}^{*}$ and $T_{M}$, we write $\theta(X)=\langle\theta, X\rangle$, and then we have $\|\phi\|^{2}\langle\theta, X\rangle=$ $\langle i \phi(\theta), i \phi(X)\rangle$. Therefore, for all $X$,

$$
\begin{gathered}
2\langle i \theta \bullet \phi, i \phi(X)\rangle+\left\langle\mathcal{D}^{A+2 i \theta} \phi, i \phi(X)\right\rangle=0, \\
\left\langle\mathcal{D}^{A+6 i \theta} \phi, i \phi(X)\right\rangle=0
\end{gathered}
$$

since $\mathcal{D}^{A+6 i \theta} \phi=\mathcal{D}^{A+2 i \theta} \phi+2 i \theta \bullet \phi$. But then, on the support of $\phi$, we must have

$$
\mathcal{D}^{A+6 i \theta} \phi=0
$$

and so we have proved

$(\alpha)$ If $A$ is the minimum point for $\mathcal{B} \phi$, and $A+2 i \theta$ is the minimum point for Alt $\mathcal{B} \phi$, then $\mathcal{D}^{A+6 i \theta} \phi=0$. 
Assume now that $A$ is the minimizing connection for $\mathcal{B} \phi$, so that, for all $X$,

$$
\left\langle\bar{\nabla}_{X}^{A} \phi, i \phi\right\rangle=0
$$

Assume also that $A+2 i \eta$ minimizes $\operatorname{Sym} \mathcal{B} \phi$ : for all $X$

$$
\begin{array}{r}
6\left\langle\bar{\nabla}_{X}^{A+2 i \eta} \phi, i \phi\right\rangle-\left\langle\mathcal{D}^{A+2 i \eta} \phi, i \phi(X)\right\rangle=0, \\
6\|\phi\|^{2} \eta(X)-\left\langle\mathcal{D}^{A+2 i \eta} \phi, i \phi(X)\right\rangle=0 \\
\langle 6 i \eta \bullet \phi, i \phi(X)\rangle-\left\langle\mathcal{D}^{A+2 i \eta}, i \phi(X)\right\rangle=0 \\
-\left\langle\mathcal{D}^{A-10 i \eta} \phi, i \phi(X)\right\rangle=0
\end{array}
$$

and so $\mathcal{D}^{A-10 i \eta} \phi=0$ on the support of $\phi$. We have obtained

( $\beta$ ) If $A$ is the minimum point for $\mathcal{B} \phi$, and $A+2$ in is the minimum point for $\operatorname{Sym} \mathcal{B} \phi$, then we have $\mathcal{D}^{A-10 i \eta} \phi=0$.

If we now combine $(\alpha)$ and $(\beta)$ above with Remark [3.10, then Theorem [3.7]will follow.

Proof of Theorem 3.8. The formula for $\xi$ is obtained by comparing the minimizing connection for $\mathcal{B} \phi$ with the vanishing connection for $\mathcal{D} \phi$. The main ingredient is formula (31) (from the Introduction; it is proved in Sco02]), combined with Lemma 3.9(A) and the suitable formula from (6). Concretely, if $\mathcal{B}^{A} \phi$ is minimal and $\mathcal{D}^{A+2 i \theta} \phi=0$, then

$$
\theta=-\frac{2}{\|\phi\|^{2}} d^{*} \sigma
$$

Fitting $\theta$ to $\xi$ from Theorem 3.7 yields the result.

Proof of Lemma 3.9(A). The minimality formula (7) applied to $\mathcal{T}=\mathcal{B} \phi$ becomes the condition that, for all $\left.\theta \in T_{M}^{*}\right|_{x}$, we have

$$
\begin{aligned}
& \left.\sum_{j, k=1}^{4}\left\langle\left(\mathcal{B}^{A_{0}} \phi\right)_{e_{j}} e_{k}, i \theta\left(e_{j}\right) \phi\left(e_{k}\right)\right\rangle\right|_{x}=0, \\
& \left.\sum_{j, k=1}^{4} \theta\left(e_{j}\right)\left\langle\left(\mathcal{B}^{A_{0}} \phi\right)_{e_{j}} e_{k}, i \phi\left(e_{k}\right)\right\rangle\right|_{x}=0 .
\end{aligned}
$$

Since that must happen for all $\left.\theta \in T_{M}^{*}\right|_{x}$, we must have, for all $j$,

$$
\begin{aligned}
\left.\sum_{k=1}^{4}\left\langle\left(\mathcal{B}^{A_{0}} \phi\right)_{e_{j}} e_{k}, i \phi\left(e_{k}\right)\right\rangle\right|_{x} & =0, \\
\left.\sum_{k=1}^{4}\left\langle e_{k} \bullet \vec{\nabla}{ }_{e_{j}}^{A_{0}} \phi, e_{k} \bullet i \phi\right\rangle\right|_{x} & =0 \\
\sum_{k=1}^{4}\left\langle\vec{\nabla} e_{e_{j}}^{A_{0}} \phi, i \phi\right\rangle_{x} & =0
\end{aligned}
$$

which means that $\left\langle\vec{\nabla}_{e_{j}}^{A} \phi, i \phi\right\rangle=0$ and thus concludes the proof of $3.9(\mathrm{~A})$.

Proof of Lemma 3.9.(B). The minimality condition for $\operatorname{Sym}^{A} \phi$ is

$$
\left.\sum_{j, k=1}^{4}\left\langle\left(\operatorname{Sym} \mathcal{B}^{A_{0}} \phi\right)_{e_{j}} e_{k},\left(\operatorname{Sym} \mathcal{B}^{A_{0}} \phi\right)_{e_{j}} e_{k}-\left(\operatorname{Sym} \mathcal{B}^{A} \phi\right)_{e_{j}} e_{k}\right\rangle\right|_{x}=0
$$

for all $\left.\theta \in T_{M}^{*}\right|_{x}$. That is,

$$
\begin{array}{r}
\left.\sum_{j, k=1}^{4}\left\langle\left(\mathcal{B}^{A_{0}} \phi\right)_{e_{j}} e_{k}+\left(\mathcal{B}^{A_{0}} \phi\right)_{e_{k}} e_{j}, i \theta\left(e_{j}\right) \phi\left(e_{k}\right)+i \theta\left(e_{k}\right) \phi\left(e_{j}\right)\right\rangle\right|_{x}=0, \\
\sum_{j=1}^{4} \theta\left(e_{j}\right)\left(\sum_{k=1}^{4}\left\langle\left(\mathcal{B}^{A_{0}} \phi\right)_{e_{j}} e_{k}, i \phi\left(e_{k}\right)\right\rangle+\left\langle\left(\mathcal{B}^{A_{0}} \phi\right)_{e_{k}} e_{j}, i \phi\left(e_{k}\right)\right\rangle_{x}\right)=0 .
\end{array}
$$


Therefore, for all $j$,

$$
\begin{aligned}
\sum_{k=1}^{4}\left\langle\left(\mathcal{B}^{A_{0}} \phi\right)_{e_{j}} e_{k}, i \phi\left(e_{k}\right)\right\rangle+\left.\left\langle\left(\mathcal{B}^{A_{0}} \phi\right)_{e_{k}} e_{j}, i \phi\left(e_{k}\right)\right\rangle\right|_{x} & =0, \\
\sum_{k=1}^{4}\left\langle e_{k} \bullet \vec{\nabla}_{e_{j}}^{A_{0}} \phi, e_{k} \bullet i \phi\right\rangle+\left.\left\langle e_{j} \bullet \vec{\nabla}_{e_{k}}^{A_{0}} \phi, e_{k} \bullet i \phi\right\rangle\right|_{x} & =0 .
\end{aligned}
$$

But $\left\langle e_{j} \bullet a, e_{k} \bullet b\right\rangle=-\left\langle e_{k} \bullet a, e_{j} \bullet b\right\rangle$ when $j \neq k$. So, for every $j$,

$$
\begin{aligned}
5\left\langle\vec{\nabla}_{e_{j}}^{A_{0}} \phi, i \phi\right\rangle-\left.\sum_{k \neq j}\left\langle e_{k} \bullet \vec{\nabla}_{e_{k}}^{A_{0}} \phi, e_{j} \bullet i \phi\right\rangle\right|_{x} & =0, \\
6\left\langle\vec{\nabla}_{e_{j}}^{A_{0}} \phi, i \phi\right\rangle-\left\langle\mathcal{D}^{A_{0}} \phi,\left.e_{j} \bullet i \phi\right|_{x_{x}}\right. & =0
\end{aligned}
$$

which concludes the proof of $3.9(\mathrm{~B})$.

Proof of Lemma 3.9 C). Minimality of Alt $\mathcal{B}^{A} \phi$ is insured by

$$
\begin{aligned}
& \left.\sum_{j \neq k}\left\langle\left(\mathcal{B}^{A_{0}} \phi\right)_{e_{j}} e_{k}-\left(\mathcal{B}^{A_{0}} \phi\right)_{e_{k}} e_{j}, i \theta\left(e_{j}\right) \phi\left(e_{k}\right)-i \theta\left(e_{k}\right) \phi\left(e_{j}\right)\right\rangle\right|_{x}=0, \\
& \sum_{j \neq k} \theta\left(e_{j}\right)\left(\left\langle\left(\mathcal{B}^{A_{0}} \phi\right)_{e_{j}} e_{k}, i \phi\left(e_{k}\right)\right\rangle-\left.\left\langle\left(\mathcal{B}^{A_{0}} \phi\right)_{e_{k}} e_{j}, i \phi\left(e_{k}\right)\right\rangle\right|_{x}\right)=0 .
\end{aligned}
$$

Then, for all $j$,

$$
\begin{array}{r}
\sum_{k \neq j}\left(\left\langle e_{k} \bullet \vec{\nabla}_{e_{j}}^{A_{0}} \phi, e_{k} \bullet i \phi\right\rangle-\left.\left\langle e_{j} \bullet \vec{\nabla}_{e_{k}}^{A_{0}} \phi, e_{k} \bullet i \phi\right\rangle\right|_{x}\right)=0, \\
3\left\langle\vec{\nabla} \vec{e}_{e_{j}}^{A_{0}} \phi, i \phi\right\rangle+\left.\sum_{k \neq j}\left\langle e_{k} \bullet \vec{\nabla}_{e_{k}}^{A_{0}} \phi, e_{j} \bullet i \phi\right\rangle\right|_{x}=0, \\
2\left\langle\vec{\nabla}_{e_{j}}^{A_{0}} \phi, i \phi\right\rangle+\left\langle\mathcal{D}^{A_{0}} \phi,\left.e_{j} \bullet i \phi\right|_{x_{x}}=0\right.
\end{array}
$$

which concludes the proof of Lemma $3.9(\mathrm{C})$.

Proof of Lemma 3.1 (and construction of $\bar{\nabla}^{A}$ and $\widetilde{\nabla}^{A}$ ). In order to prove 3.1. we need to clearly explain how $\nabla$ and $A$ induce the connections $\bar{\nabla}^{A}$ and $\widetilde{\nabla}^{A}$. In tune with the rest of the paper, we will use the language of quaternions.

The group $S O(4)=\mathbb{S}^{3} \times \mathbb{S}^{3} / \pm 1$ acts on $\mathbb{R}^{4} \approx \mathbb{H}$ by $\left[\xi_{+}, \xi_{-}\right] \cdot v=\xi_{+} v \xi_{-}^{-1}$. Its Lie algebra is $\mathfrak{s o}(4)=\operatorname{Im} \mathbb{H} \oplus \operatorname{Im} \mathbb{H}$ and its adjoint action on $\mathbb{R}^{4}$ is $\left(q_{+} \oplus q_{-}\right) \cdot v=$ $q_{+} v-v q_{-}$. Therefore, any $g$-metric connection $D$ on $T_{M}$ can be written locally as $D v=\partial v+b_{+} v-v b_{-}$for suitable local 1-forms $b_{ \pm} \in \Gamma\left(T_{M}^{*} \otimes \operatorname{Im} \mathbb{H}\right)$. In particular, we write the Levi-Cività connection $\nabla$ on $T_{M}$ locally as $\nabla v=\partial v+\mathfrak{a}_{+} v-v \mathfrak{a}_{-}$, for some $\mathfrak{a}_{ \pm} \in \Gamma_{\text {loc }}\left(T_{M}^{*} \otimes \operatorname{Im} \mathbb{H}\right)$. The connection $D$ induces connections on all tensor bundles of $M$, and in particular on $\Lambda^{ \pm}\left(T_{M}^{*}\right)$; the latter can be written locally as $\left.D\right|_{\Lambda^{ \pm}} f=\partial f+b_{ \pm} f-f b_{ \pm}$.

The group $U(1)=\mathbb{S}^{1}$ acts on $\mathbb{C}$ by left multiplication, and its Lie algebra is $\mathfrak{u}(1)=i \mathbb{R}$. Thus, any unitary connection on the complex line bundle $K^{*}$ can be written locally as $A z=\partial z+2 i \alpha z$, for some suitable $\alpha \in \Gamma_{l o c}\left(T_{M}^{*}\right)$.

The group $U(2)=\mathbb{S}^{1} \times \mathbb{S}^{3} / \pm 1$ acts on $\mathbb{C}^{2} \approx \mathbb{H}$ by $[\lambda, \xi] \cdot w=\lambda w \xi^{-1}$. Its Lie algebra is $\mathfrak{u}(2)=i \mathbb{R} \oplus \operatorname{Im} \mathbb{H}$ and its adjoint action on $\mathbb{C}^{2}$ is $(i \ell \oplus q) \cdot w=i \ell w-w q$. Hence, any unitary connection on $\mathcal{W}^{ \pm}$can be written locally as $D w=\partial w+i \beta w-w \delta$ for some suitable $\beta \in \Gamma_{l o c}\left(T_{M}^{*}\right)$ and $\delta \in \Gamma_{l o c}\left(T_{M}^{*} \otimes \operatorname{Im} \mathbb{H}\right)$.

The group $\operatorname{Spin}^{\mathbb{C}}(4)=\mathbb{S}^{1} \times \mathbb{S}^{3} \times \mathbb{S}^{3}$ has Lie algebra $\mathfrak{s p i n}^{\mathbb{C}}(4)=i \mathbb{R} \oplus \operatorname{Im} \mathbb{H} \oplus$ $\operatorname{Im} \mathbb{H}$. The canonical maps $\operatorname{Spin}^{\mathbb{C}}(4) \rightarrow S O(4), \operatorname{Spin}^{\mathbb{C}}(4) \rightarrow \mathbb{S}^{1}$, and the two maps $\operatorname{Spin}^{\mathbb{C}}(4) \rightarrow U(2)$ have the Lie algebra versions

$$
\begin{aligned}
& \mathfrak{u}(1) \longleftarrow \mathfrak{s p i n}^{\mathbb{C}}(4) \quad \longrightarrow \quad \mathfrak{s o}(4) \quad \mathfrak{s p i n}^{\mathbb{C}}(4) \quad \longrightarrow \mathfrak{u}(2)
\end{aligned}
$$

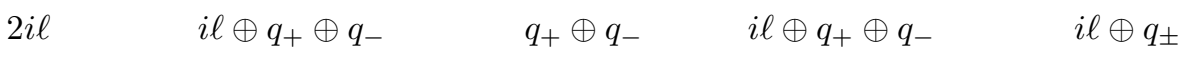


The consequence is that, if one chooses a unitary connection $A z=\partial z+2 i \alpha z$ on $K^{*}$, then, combining with the Levi-Cività connection $\nabla$ and using the above maps, $A$ determines unique unitary connections on $\mathcal{W}^{ \pm}$, namely $\bar{\nabla}^{A} w=\partial w+i \alpha w-w \mathfrak{a}_{+}$ on $\mathcal{W}^{+}$, and $\widetilde{\nabla}^{A} w=\partial w+i \alpha w-w \mathfrak{a}_{-}$on $\mathcal{W}^{-}$.

The fact that $\nabla$ and $\widetilde{\nabla}^{A}$ share the coefficient-form $\mathfrak{a}_{-}$can be expressed as $\left.\nabla\right|_{\Lambda^{-}}=$ $\left.\widetilde{\nabla}^{A}\right|_{\Lambda^{-}}$. Indeed the bundle $\Lambda^{-}\left(T_{M}^{*}\right)$ has model-fiber $\operatorname{Im} \mathbb{H}$ with structure group $S O(3)=\mathbb{S}^{3} / \pm 1$ acting as $[\xi] \cdot a=\xi a \xi^{-1}$. The Lie algebra $\mathfrak{s o}(3)$ is $\operatorname{Im} \mathbb{H}$ and its adjoint action is $h \cdot a=h a-a h$. The cocycle of $\Lambda^{-}$is induced from the cocycle of $T_{M}$ via the map $S O(4) \rightarrow S O(3),\left[\xi_{+}, \xi_{-}\right] \longmapsto\left[\xi_{-}\right]$. Thus, a connection $D v=\partial v+b_{+} v+v b_{-}$induces on $\Lambda^{-}$the connection $\left.D\right|_{\Lambda^{-}} a=\partial a+b_{-} a-a b_{-}$.

Assume that $D$ is a $g$-metric connection on $T_{M}$ that has $\left.D\right|_{\Lambda^{-}}=\left.\nabla\right|_{\Lambda^{-}}$. Then $D v=\partial v+b_{+} v-v \mathfrak{a}_{-}$. If further $D$ is $\mathbb{C}$-linear, then the quaternionic-imaginary form $b_{+}$must in fact be just complex-imaginary, and thus $b_{-}=i \alpha$ for some $\alpha \in \Gamma_{l o c}\left(T_{M}^{*}\right)$. But it can be verified that these forms $\alpha$ define a unitary connection $A z=\partial z+2 i \alpha z$ on $K^{*}$ (compare with Remark 3.2).

\section{ACKNOWLEDGMENTS}

We wish to thank R. Kirby for help and support during sometimes difficult moments. We also wish to thank D. Freed and C. Taubes for sharp comments on an earlier version of this paper.

\section{REFERENCES}

[AHS78] M. F. Atiyah, N. J. Hitchin, and I. M. Singer, Self-duality in four-dimensional Riemannian geometry, Proc. Roy. Soc. London Ser. A 362 (1978), no. 1711, 425-461. MR 80d:53023

[Akb96] Selman Akbulut, Lectures on Seiberg-Witten invariants, Turkish J. Math. 20 (1996), 95-119. MR 97g:57038

[Bär97] Christian Bär, On nodal sets for Dirac and Laplace operators, Comm. Math. Phys. 188 (1997), no. 3, 709-721. MR 98g:58179

[Don96] S. K. Donaldson, The Seiberg-Witten equations and 4-manifold topology, Bull. Amer. Math. Soc. (N.S.) 33 (1996), no. 1, 45-70. MR 96k:57033

[LM89] H. Blaine Lawson, Jr. and Marie-Louise Michelsohn, Spin geometry, Princeton University Press, Princeton, NJ, 1989. MR 91g:53001

[Mor96] John W. Morgan, The Seiberg-Witten equations and applications to the topology of smooth four-manifolds, Princeton University Press, Princeton, NJ, 1996. MR 97d:57042

[Sco02] Alexandru Scorpan, Nowhere-zero harmonic spinors and their associated self-dual 2forms, Commun. Contemp. Math. 4 (2002), no. 1, 45-63. MR 2003c:53064

[Tau95] Clifford Henry Taubes, The Seiberg-Witten and Gromov invariants, Math. Res. Lett. 2 (1995), no. 2, 221-238. MR 96a:57076

[Tau00] Seiberg-Witten and Gromov invariants for symplectic 4-manifolds, International Press, Somerville, MA, 2000, Edited by Richard Wentworth. MR 2002j:53115

[Wit94] Edward Witten, Monopoles and four-manifolds, Math. Res. Lett. 1 (1994), no. 6, 769796. MR 96d:57035

Department of Mathematics, University of California Berkeley, 970 Evans Hall, Berkeley, California 94720

E-mail address: scorpan@math.berkeley.edu

Current address: Department of Mathematics, University of Florida, Gainesville, Florida 32611-8105

E-mail address: ascorpan@math.ufl.edu

$U R L:$ www.math.ufl.edu/ scorpan 